\title{
¿De la Primavera al Verano Árabe?
}

\author{
Editado por/Edited by: David Vasquez \\ Recibido/Received: 9/11/2014. Aceptado/Accepted: 16/05/2015 \\ Publicado en línea/Published on Web: 30/04/2015
}

\author{
Flavio Carrera \\ Universidad San Francisco de Quito, Colegio de Ciencias Sociales y Humanidades COCISOH \\ Diego de Robles y Vía Interoceánica, Quito, Ecuador
}

Correo Electrónico: flavio.carrera@estud.usfq.edu.ec

\section{Resumen}

Este artículo explora los factores políticos (participación ciudadana), sociales (capital social), educativos (alfabetismo) y económicos (infraestructura e inversión) que se considera han permitido la consolidación de la democracia en Túnez y una parcial de la misma en Egipto. Para sustentar estas afirmaciones se han empleados índices como el Democratic Index, Doing Business o Global Competitiveness, mismos que han sido de apoyo para los factores antes mencionados. Los argumentos de que la consolidación democrática de Túnez, y la parcial de Egipto, resultan del capital social, alfabetismo, infraestructura o participación ciudadana, están basadas principalmente en procesos similares iniciados en distintas latitudes, demostrando la validez de los mismos.

Palabras clave: democracia, Primavera Árabe, Túnez, Egipto, capital social, alfabetismo, infraestructura, participación ciudadana.

\section{Abstract}

This article explores the political (civic participation), social (social capital), education (literacy) and economic factors (infrastructure and investment) considered have allowed the consolidation of democracy in Tunisia and a partial consolidation of the same in Egypt. To support these arguments we have used some index like the Democratic Index, Doing Business and Global Competitiveness, giving reinforcement to the ideas and factors aforementioned. The arguments that democratic consolidation in Tunisia, and the partial consolidation of Egypt, are a result of social capital, literacy, infrastructure or public participation are based mainly on similar processes initiated in different latitudes, demonstrating the validity of them.

Keywords: democracy, Arab Spring, Tunisia, Egypt, social capital, literacy, infrastructure, civic participation.

A

lo largo de los últimos 3 años y medio, hemos visto un nuevo movimiento político, social y económico, que ha surgido en varios países de Medio Oriente, que fue calificado como Primavera Árabe o Arab Spring. Este proceso surge en Túnez a finales de 2010, y su objetivo fue la búsqueda de mayores libertades políticas, sociales y económicas para toda la población, acabando con gobiernos autoritarios que manejaron por más de 30 años. Dentro del contexto político, social y económico este movimiento trajo como derivación tres panoramas con distintos resultados. Se presenta, por un lado, un caso exitoso de consolidación democrática, Túnez, que acaba de celebrar sus segundas elecciones hace cinco meses. Egipto es un caso de parcial éxito, habiendo tenido un presidente elegido democráticamente, quien por abusos y mal gobierno fue retirado del poder con un golpe de Estado encabezado por las fuerzas armadas. Finalmente tenemos a Libia o Siria, países que en la actualidad viven guerras civiles con marcadas divisiones internas.

A partir de este contexto, el presente artículo busca explorar los factores que han conducido a diferentes consecuencias en los países que vivieron este proceso político y social. Se considera que la Primavera Árabe ha llevado a dos escenarios: la consolidación de un sistema democrático en el gestor de este movimiento, Túnez, que contrasta con una incompleta consolidación de la democracia en Egipto, abrien- 
do paso a una situación peor a la vivida durante el gobierno de Hosni Mubarak, presidente derrocado tras las manifestaciones. En el presente artículo se busca demostrar y analizar los factores que han permitido la consolidación democrática en Túnez y que han estado ausentes en Egipto. Antes de pasar al análisis es conveniente explicar el contexto.

\section{Túnez}

Es el Estado que inició este movimiento, sacando del poder a Zine el Abidie Ben Ali luego de 23 años en el cargo presidencial. En 2011 se convocaron elecciones para elegir a un gobierno democrático celebradas en octubre del mismo año, cuyo ganador fue Ennahda (partido islamista moderado, conocido como Partido del Renacimiento) con un 37\% (Al Jazeera, 2011), aliándose con el Congress for the Republic (CPR) que obtuvo 8,71\% y Ettakatel con el $7.03 \%$ (dos partido seculares). El resultado fue la conformación de un gobierno de coalición entre islamistas moderados y grupos seculares.

Se formó un gobierno de tecnócratas, al ser aprobada la Constitución que promulgaba valores de libertad, justicia y democracia. Posteriormente, en octubre de 2014, se celebraron elecciones parlamentarias exitosas, con mayoría del partido secular Nidaa Tounes (ocupando 86 curules), en coalición a los islamistas moderados de Ennahda (ocupando 69 curules) (Reuters, 2014).

\section{Egipto}

Luego de 29 años en el poder, los movimientos sociales (Muslim Brotherhood, Sociedad Civil, Mujeres y jóvenes) sacaron del poder a Hosni Mubarak, se convocaron elecciones entre Mayo y Junio de 2012, siendo elegido Mohammed Morsi de Freedom and Justice (partido islamista parte de la Hermandad Musulmana) con el 51,7\%, frente al Ahmed Shafik con un 48,3\% de los votos. En este contexto no se formó un gobierno de coalición basados en la visión de la mayoría otorgada por las urnas, donde muchos deciden sobre pocos (Weaver \& Whitaker, 2012).
En julio de 2013, Morsi fue derrocado tras un golpe de estado encabezado por el Mariscal Abdel Fatah al-Sisi, como respuesta a los altos niveles de protesta, con un $61 \%$ de los egipcios insatisfechos con el mismo (Khazan, 2013). El derrocamiento trajo consigo consecuencias en el ámbito político y social similares a los de los años de represión durante el gobierno de Mubarak.

El proceso de consolidación democrática vivido en Túnez, frente al fallido intento en Egipto, están reflejados en índices como el Freedom in the World elaborado por Freedom House (Freedom House, 2015), en el cual para el año 2014 (último reporte) encontramos a Túnez dentro de la categoría de Países Libres con una calificación de 79/100, como reflejo de todos los espacios democráticos abiertos (elecciones, participación etc.) frente a un Egipto considerado No Libre cuya calificación es de 26/100, reflejo de los gobiernos represivos de Morsi y Al-Sisi.

En cuanto a la calificación de su democracia, en el Democratic Index elaborado por The Economist (The Economist, 2014), para el reporte de 2013 Túnez se ubica en la posición 90 considerado como un Régimen Híbrido, y Egipto en el puesto 135 como un Régimen Autoritario y Represivo. Esto no solo se refleja en índices. Mientras en Túnez se logra una mayor consolidación democrática con una participación más activa de diversas facciones y grupos sociales, en Egipto existe todo tipo de represión (encarcelamiento, juicios políticos, expulsión del país etc.) contra las voces disidentes que hacen uso de su derecho de expresión. Esto se evidenció en los hechos del 25 de enero de 2015, día que se celebraba un año más de la revolución que sacó del poder a Mubarak, con un saldo trágico de más de 11 personas fallecidas entre ellas la reconocida activista, Shaima al Sabbagh (líder del partido Alianza Popular Socialista), quien fue tiroteada por las fuerzas armadas egipcias (Monzón, 2015). En otras palabras, estamos frente a dos panoramas completamente distintos por un lado un pleno estado de derecho con participación y pluralidad frente a un estado sin derechos en el que cualquier voz disidente es acallada. Reflejándose lo mismo en los dos índices antes mencionados.A continuación se analizan los diversos factores que han contribuido a la consolidación en Túnez, en comparación a Egipto. 


\section{Participación Política}

En primer lugar, en Túnez se evidencia pluralidad e inclusión en los diversos poderes del Estado. Ejemplo de ello, en la conformación de la Asamblea Constituyente y posterior composición del gobierno. En el primer caso el partido más votado fue Ennahda (con un 37\%), que junto a CPR (con 8,71\%) y Ettakatel (con 7,03\%) establecieron una coalición de trabajo (Abdessalem, 2014) para sacar adelante el proyecto de Constitución sumado más tarde a la formación de un gobierno con facciones islamistas moderadas (Ennahda) y seculares (CPR-Ettakatel entre otros). Más tarde los partidos cedieron para formar un gobierno de tecnócratas, quienes dirijan el Estado con un objetivo básico: encaminar a Túnez hacia una democracia duradera.

Este pluralismo permitió elaborar, por un lado, una Constitución donde se respete y se rija a base de la libertad y la justicia en un marco democrático, con un alto porcentaje de secularización de la misma, respetando todos los derechos ganados a lo largo del tiempo para minorías religiosas y sociales (mujeres). Como dato, en la sociedad tunecina existe un apoyo significativo a que mujeres y hombres compartan los mismos derechos: según obtenidos del Pew Research Global Attitudes Project, para el año 2013 un 63\% de la población está a favor de igualdad de derechos (Pew Research Center, 2013).

Por otro lado, el pluralismo permitió mantener una transición democrática para consolidarla, con las elecciones de octubre 2014 (Markey \& Amrara, 2014). Aunque triunfaron facciones seculares, éstas han abierto canales de comunicación con los islamistas moderados para continuar el proceso de trabajo en equipo que iniciaron éstos últimos en 2011 (Ottaway, 2014). Se incluyen entre estos canales el trabajo en conjunto en proyectos de ley y participación en la conformación del gabinete ministerial con cuota política en ella. Esto permite tener un primer acercamiento para comprender la consolidación y relativo éxito de la democracia tunecina.

La participación política, es otro de los componentes empleados para determinar los niveles de vínculos sociales (social bonds) existentes. Al recor- dar las elecciones de 2014, que en Túnez fueron para la elección del parlamento y en Egipto para la elección de un nuevo presidente, se concluye que los niveles de participación ratifican la calidad de relaciones existentes, porque demuestran el compromiso de la población con el proceso iniciado. Este es sin duda un elemento valioso para la conformación de las instituciones que la conforman. Para Túnez la participación registró el 57.7\% (La Vanguardia, 2014) pero en el caso de Egipto fue necesario abrir las urnas durante 3 días por la falta de votantes, alcanzando, finalmente un $46,8 \%$ (Berghe, 2014). Los datos antes presentados demuestran el nivel de compromiso que tienen estas dos sociedades con el proceso político que han instaurado, y ratifica el punto de que en Túnez se ha logrado consolidar la democracia por un mayor compromiso de la sociedad, frente al menos exitoso proceso trabajado en Egipto. Con este escenario se propicia el desarrollo no solo de relaciones sociales de mucha mayor confianza entre los miembros de la sociedad sino también se traduce en la conformación del Estado y su desarrollo.

La razón por la cual la menor participación política debilita cualquier proceso democrático es por la necesidad de que exista un consenso con objetivos en común; es decir, buscando sobreponer los intereses personales o de una facción de la población a los intereses de todo el Estado (algo que ocurre en Túnez donde se busca establecer una democracia, con la conformación del gobierno tecnocrático). Además, se debilita debido a que internamente existen conflictos entre las diversas facciones, como por las distintas etnias existentes (división poblacional), cuyo resultado es una situación de caos, que lo único que hace es detener cualquier proceso de transformación política que viva un país.

Esto se puede observar claramente hoy en día en Egipto. Al haber derrocado a Mubarak, se convocó a elecciones y una de las facciones, los Hermanos Musulmanes, las ganaron. Al llegar al poder buscaron instaurar políticas fuertemente ligadas con el Islam (con altos niveles de apoyo de la carta magna como las leyes a las enseñanzas del Corán), con más del $60 \%$ de apoyo popular y con más de $75 \%$ de la población viendo con buenos ojos la intervención de líderes religiosos en la política (Pew Research Center, 2013). También buscaron atacar a otras partes de la 
sociedad, lo que impulsó protestas, asesinatos e inclusive derrocamiento del primer gobierno electo en toda la historia de Egipto (el de Mohammed Morsi). Así se generó una colectividad llena de conflictos entre diversas partes de la misma, que resultará difícil de unificar.

A esto se suma que, al acceder al poder, Morsi nunca decidió trabajar en un gobierno de coalición política como en Túnez sino todo lo contrario: atacó e invisibilizó a la oposición, creando una constitución altamente islamista, sectaria, con aprobación de más del 58\% de la población, quienes apoyan una constitución conectada con el Islam (Pew Research Center, 2013); generando gran oposición interna, repitiendo los vicios que buscaban en el fondo evitar.

Esto con el poco o nulo conocimiento por parte de la mayoría de la población de los procesos formales o administrativos que se deben seguir en democracia, debido a que nunca ha participado de manera directa en la conformación de un gobierno de carácter democrático, con Egipto cómo caso donde por más de 60 años no han tenido un gobierno elegido democráticamente afecta en su participación y consolidación como sociedad.

\section{Infraestructura}

En segundo lugar, en cuanto al tema de la infraestructura, Túnez cuenta con mejores niveles de infraestructura frente a Egipto, lo cual se evidencia con varios índices. Uno de ellos es el Doing Business del World Bank Group, en la cual Túnez se ubica en el lugar 60 del mundo y 5 de la región, frente a Egipto que ocupa la posición 112 mundial y 11 regional. Este índice no solo califica la apertura económica, sino la facilidad de recursos e infraestructura existente en los países para el desarrollo industrial en ellos (World Bank Group, 2014).

El tema de la infraestructura es ratificado con los datos del Global Competitiveness del World Economic Forum, donde Túnez ocupa la posición 79 con $3.8 / 7$, uno de los más altos en relación a su zona geográfica, frente a la posición 100 con 3.2/7 que ocupa Egipto, existiendo una mayor posibilidad de desarro- llo en un país que presente mejores condiciones de infraestructura y seguridad para inversiones extranjeras como es el caso de Túnez frente a Egipto.

\section{Educación}

En cuanto al tema educativo los niveles de alfabetismo en la sociedad tunecina frente a la egipcia son mayores en $14.4 \%$, lo que permite inferir que los niveles de enseñanza en Túnez son mucho mayores que los existentes en Egipto. Esto es reafirmado en el índice educativo del Global Competitiveness, que califica la calidad y competitividad de los estados y sus economías en varios campos, enfocados sobre todo a el tema de rendición de cuentas, índices de corrupción e infraestructura elaborado por el World Bank Group (World Bank Group, 2014). En este análisis, aplicado en 144 países, Túnez se ubica en el puesto 54 con 5.4 puntos, frente a Egipto, en el puesto 106 con 4.3 puntos.

Lo anterior da mayor validez al argumento de que el factor educativo juega un rol importante en el proceso de consolidación democrática. La Tabla 1 a continuación muestra los niveles de alfabetismo en Túnez, Egipto y varios de sus países vecinos. Puede verse una sustancial diferencia a favor de Túnez.

TABLA 1: Niveles de alfabetismo comparado con Túnez

\begin{tabular}{c|c} 
País & Alfabetismo \\
\hline Libia & $94,2 \%$ \\
\hline Jordania & $93.4 \%$ \\
\hline Argelia & $91,8 \%$ \\
\hline Líbano & $89,6 \%$ \\
\hline Túnez & $88.3 \%$ \\
\hline Egipto & $73,9 \%$ \\
\hline Sudán & $71,9 \%$
\end{tabular}

Datos obtenidos de estudio de UNICEF a 2013. (UNICEF, 2013)

En cuanto a la educación a mujeres, Túnez cuenta con un porcentaje más alto de población feme- 
nina educándose, alcanzando el $80 \%$ frente al $65 \%$ de la población egipcia, lo que denota mayor apertura hacia una educación inclusiva, igualitaria, que es reflejada en leyes de igualdad de género y condena a cualquier ataque por género o etnia.

\section{Capital Social y Vínculos Sociales}

Otro argumento de por qué Túnez ha alcanzado un proceso exitoso hacia la democracia parte de la evidencia anterior unida al concepto de Capital Social (Social Capital) de Putnam. El concepto radica en la creación y/o existencia de normas de reciprocidad general y canales de compromiso social, que impulsan la cooperación y confianza social, reduciendo la incertidumbre y el deseo de vencer a otros, como un modelo de futura cooperación (Putnam, 1993). Enfocados en tres aspectos como son la seguridad, participación política y niveles de corrupción, que permiten la existencia de vínculos sociales (social bonds) entre las personas de una sociedad, que permiten lograr confianza y cooperación mutua; estos son mucho más fuertes en Túnez, lo que ha determinado su notable éxito democrático, el mismo que no goza la población egipcia.

Importantes elementos del Capital Social, son los diversos tipos de organizaciones como sindicatos, asociaciones cívicas y sociedades de profesionales (médicos, abogados, ingenieros etc.). Sumado a ello tener un buen número de organizaciones sociales más las condiciones antes mencionadas, que tiene Túnez se traducen en mejores relaciones sociales, reflejado en niveles de participación política, como más de $57 \%$ en las elecciones de 2014 (La Vanguardia, 2014) que muestran el compromiso con el proceso democrático. Asimismo se suma tener sindicatos que a pesar de ser perseguidos y censurados, trabajan comprometidos con el desarrollo de Túnez, como es la Union Général Tunisienne du Travail (UGTT) (Trabelsi, 2012).

En cuanto a los vínculos sociales que contribuyen de manera directa al tema del Capital Social, partimos del tema de la seguridad. Según datos obtenidos de Global Competitiveness, Túnez ocupa la posición 113 con 3.8/7, y Egipto la posición 140 con 2.9/7 puntos (World Economic Forum, 2014). En este índice se midió no solo el tema de seguridad para es- tablecer negocios, sino la seguridad jurídica, personal y confianza entre personas. Este primer factor permite ver que los niveles de seguridad son mucho mayores de un país a otro, lo que impulsa una mejor relación entre miembros de la sociedad, con un nivel más alto de confianza entre miembros, formándose los vínculos sociales necesarios para mantener un capital social importante.

Un elemento de inseguridad en una zona con altos conflictos armados por los fanatismos religiosos, es la existencia de grupos islamistas radicales (ISIL, Boko Haram, Al Qaeda etc.) y el nivel de preocupación por parte de la población de la existencia y desarrollo de los mismos. Los datos obtenidos al respecto ratifican que en Túnez existen mejores relaciones sociales frente a Egipto, porque en Túnez existe un $60 \%$ de la población que considera preocupante la presencia de estos grupos, frente a un 34\% en Egipto (Pew Research Center, 2013). Cuando la población es consultada acerca de que si estos grupos deberían ser una preocupación mundial, los datos ratifican lo antes mencionado porque un $64 \%$ de tunecinos los ven con profunda preocupación, frente a un $41 \%$ de egipcios (Pew Research Center, 2013). Sin embargo, para un $43 \%$ de egipcios este es un tema de menor importancia frente a un $13 \%$ de tunecinos. En el tema de seguridad se ve que existen mejores relaciones sociales en Túnez que en Egipto.

Un factor que es determinante dentro de la definición del capital social y de los elementos ya analizados es el rol de las fuerzas armadas, que en el caso de Túnez no han intervenido en los procesos políticos y siempre han estado supeditados a las decisiones civiles. Aquí se establece un contraste en relación a sus vecinos porque el papel de las fuerzas armadas como "garantes de las democracia" ha sido empleado para ser ellos quienes gobiernen a largo plazo los estados. Sin embargo, en el caso de Túnez han tenido la costumbre de respetar el orden democrático (Abdessalem, 2014) y seguir los preceptos del poder ejecutivo e intereses del país.

En cuanto a Egipto, que no ha alcanzado un proceso exitoso hacia la consolidación de una democracia, el panorama es completamente distinto: no tenemos una sociedad civil vigorosa (donde solo $42 \%$ de la población está a favor de entregar los mismos derechos a mujeres y minorías) (Pew Research Cen- 
ter, 2013), con sindicatos regidos por el poder ejecutivo coartando cualquier espacio de verdadera discusión, participación y libertad.

Esto también fue reproducido en contra de Morsi y la Hermandad Musulmana por al-Sisi (Feldam, 2013), con toda la división interna ya existente tras la salida de Mubarak. Dentro de esta persecución de los dos gobiernos se acabó con la independencia y autonomía de los sindicatos laborales, industriales y sociales en Egipto, por considerarles polos de oposición y puntos de reunión anti-gubernamental, práctica del régimen de Mubarak, que fue seguida por los dos gobiernos (Trabelsi, 2012). Así se terminó con todo tipo de capital social, donde hay grupos que participen activamente en la actividad política independientemente de qué grupo étnico o partido sean. A esto sumamos los bajos niveles de participación política, demostrada en la apatía, donde solo un $46 \%$ de la población sufragó, lo que obligó a que las elecciones fueran de tres días (Berghe, 2014).

Con un capital social disminuido (bajo número de organizaciones sociales, dificultando el desarrollo y las relaciones sociales del país) y poco desarrollo de relaciones sociales o social bonds con mayor confianza entre los miembros de la sociedad (fuertemente marcada por las divisiones de carácter religioso como étnico entre suníes y chiitas), la estructuración del estado y su desarrollo se ven dificultadas, porque existe un menor número de participantes en la política (Zakaria, Can Arab countries be real democracies?, 2014), quienes crean el sistema político a base de sus intereses, excluyendo a un buen número de la población.

En el caso egipcio, esto ocurrió en la elaboración de la Constitución, donde se impuso la voluntad de Morsi y su partido, sin participación de los partidos seculares. Una constitución altamente dogmatizada en el Islam, con ataques directos a minorías existentes, lo que generó un ambiente nada democrático y de alta persecución que los propios autores de la constitución sufrieron al subir al poder Al-Sisi luego de golpe de Estado y "elecciones democráticas".

En este contexto, el rol de las fuerzas armadas egipcias es completamente distinto del de las tuneci- nas, pues en Egipto son una de las instituciones más respetadas por cerca del 60\% (Pew Research Center, 2014), que les tienen más confianza a otras instituciones. Las fuerzas armadas han estado participando activamente en la política desde 1952, y la gran diferencia con Túnez es que no se rigen de manera directa de las órdenes del poder ejecutivo. Juegan un papel independiente como fue evidente en las protestas en 2013 (Zakaria, Arab Spring's hits and misses, 2013), que terminaron con el primer gobierno democrático en la historia egipcia, como respuesta a los abusos. Luego, se instauró un sistema político represivo mucho más fuerte que en la época de Mubarak con juicios iniciados a Morsi y los líderes de la Hermandad Musulmana y a los periodistas de Al Jazeera (Al Jazeera, 2014), lo que reduce cualquier posibilidad real de alcanzar y consolidar la democracia.

A través de este artículo se ha explorado las razones por las cuales dos países que iniciaron un proceso similar obtuvieron resultados distintos. En el caso de Túnez las condiciones sociales y económicas son mucho más favorables porque existe una homogeneidad en la sociedad, sumada a que los niveles de educación en su población son altos y cuentan con infraestructura que permite el desarrollo económico y social de la población. Además, el nivel de clase media (Goldstein, 2011) es significativo, siendo ellos los motores de la economía tunecina, así como los actores políticos.

Como sugiere Mohammed Ghannouchi, líder de partido Ennahda y Primer Ministro durante el gobierno de Ben Ali, esto contribuye de manera directa a la consolidación democrática, porque las condiciones sociales para el desarrollo social están dadas a base de una buena infraestructura necesaria para la economía (Masoud, 2014), seguido de niveles educativos altos, con homogeneidad en la sociedad, sin divisiones sectarias o religiosas. Sumado a grupos islamistas moderados, quienes respetan a minorías sociales con una apertura hacia la participación de mujeres en la economía y comprometidos con un desarrollo democrático del país. Todo esto permite alcanzar una estabilidad democrática, gracias a la sociedad vigorosa existente en ella. 
En cuanto a la situación en Egipto, y a por qué no se ha logrado consolidar la democracia en ese país, se propone las razones siguientes. Primero, los conflictos religiosos y étnicos dentro de la sociedad egipcia, o lo que algunos autores han llamado los conflictos sectarios, han detenido intentos de instaurar nuevas estructuras y procesos. A raíz del derrocamiento del antiguo sistema, muchas facciones de la sociedad surgieron buscando sus reivindicaciones políticas, religiosas y sociales que no fueron logradas, durante el gobierno autoritario de Mubarak. Inclusive fueron perseguidos por pertenecer a otra facción del islam (Yihadistas y Musulmanes no radicales) (Hardy, 2013) o ser de otra etnia (Suní y Chií). Es aquí donde surgen los duros problemas sectarios, aunque por medio de movimientos como los Hermanos Musulmanes (Muslim Brotherhood) con su partido Freedom and Justice buscan su lugar en la sociedad, oponiéndose muchas veces a procesos de secularización de la sociedad, a través de la imposición de su doctrina.

Luego se da la ausencia de una clase media tan amplia (Zayed, 2014) como la tunecina, que pudiera ser un motor económico de la sociedad, la falta de infraestructura, la alta dependencia respeto de una actividad económica como el turismo, el hecho que los niveles educativos son menores, y el poco o nulo conocimiento por parte de la mayoría de la población de los procesos formales o administrativos que se deben seguir en democracia, porque nunca ha participado de manera directa en la conformación de un gobierno de carácter democrático. Tenemos el caso de muchos jóvenes que han participado en los movimientos de la Plaza de Tahrir, que nunca han vivido en gobiernos democráticos, si tomamos como punto de partida los últimos 60 años, donde militares han alternado en el poder.

Todo este desconocimiento que sufre la sociedad egipcia se traduce en un primer lugar en el desafío de establecer un nuevo sistema político que incluya las diversas posiciones políticas y religiosas existentes en el país, es decir crear una democracia participativa e inclusiva; y en segundo lugar, de elaborar todo tipo de políticas que permitan alcanzar los objetivos que meses antes habían presentado como base del movimiento (Gladstone, 2013). Debido a este desconocimiento, sumado al fuerte sectarismo y división interna, se ha dificultado la consolidación de un sistema democrático, inclusivo y participativo como el de Túnez y otras democracias.

Como se presentó, Túnez ha logrado por la vía electoral un sistema democrático confiable, plural y equilibrado, donde se respetan libertades, derechos (de minorías y mayorías) y espacios a todas las facciones de la sociedad sin importar su afiliación política o religiosa. Del otro lado se encuentra Egipto, que inició su vida democrática post-revolución con elecciones, aunque en el camino su gobernante apostara por estrategias sectarias similares a las que se buscaba erradicar, teniendo como resultado una situación adversa.

El proceso de consolidación democrática depende de muchos elementos: alfabetismo, clase media, infraestructura, social capital, etc. Junto a elecciones y líderes dispuestos a trabajar en coalición, cediendo espacios se obtienen grandes resultados. Ese es el caso de Túnez, que ha logrado no solo ingresar del autoritarismo a la democracia sino también empezar su proceso de consolidación, con un sistema abierto a la pluralidad, diversidad y espacio a todo tipo de grupos de la sociedad. Se puede observar de igual forma todo lo que no permite lograr una democracia estable, con Egipto como ejemplo.

\section{Referencias}

Abdessalem, R. (2014). Tunisia: A pioneer of Arab Democracy. Al Jazeera. Recuperado desde http://www.aljazeera.com/indepth/opinion/2014/01/tunisia-pioneer-arab-democracy-2014117827634794.html

Al Jazeera. (2011). Tunisia Election. Recuperado desde http://www.aljazeera.com/indepth/spotlight/2011tunisiaelection

Al Jazeera. (2014). Egypt sentences Al Jazeera journalist. Recuperado desde http://www.aljazeera.com/ pressoffice/2014/10/al-jazeera-journalist-sentenced-egypt-20141014104613955831.html

Berghe, A. V. (2014). Baja participación y desencanto juvenil en elecciones de Egipto. Inter Press Service. Recuperado desde http://www.ips- 
noticias.net/2014/05/baja-participacion-y-desencanto-juvenil-en-elecciones-de-egipto/

Feldam, N. (2013). Democracy is just a dream in Egypt. Bloomberg View. Recuperado desde http://www.bloombergview.com/articles/2013-08-14/democracy-is-just-adream-in-egypt

Freedom House. (2015). Freedom in the World. Recuperado desde https://www.freedomhouse.org/ report-types/freedom-world

Gladstone, R. H. (2013). Arab Spring Countries Find Peace is Harder than Revolution. The New York Times. Recuperado desde http://www. nytimes.com/2013/08/15/world/middleeast/ egypt-bloodshed-may-be-ill-omen-for-broader-region.html?smid $=$ tw-share \&_r $=0$

Goldstein, E. (2011). A Middle-Class Revolution. Foreign Policy. Recuperado desde http://www. foreignpolicy.com/articles/2011/01/18/a_middle_class_revolution

Hardy, R. (2013). Democracy or Disorder? The four lessons of the Arab Spring. $B B C$. Recuperado desde http://www.bbc.co.uk/news/world-middle-east-23266790

Khazan, O. (2013). Why Egyptians are so unhappy with Morsi, in one chart. The Atlantic. Recuperado desde http://www.theatlantic.com/international/archive/2013/07/why-egyptians-areso-unhappy-with-morsi-in-one-chart/277511/

La Vanguardia. (2014, Noviembre 23). Participación en Túnez llega al 57,73\% poco antes de cierre de los colegios. Recuperado desde http://www.lavanguardia.com/ politica/20141123/54420641298/participacion-en-tunez-llega-al-57-73-poco-antes-decierre-de-los-colegios.html

Masoud, T. (2014). Counting Islam: Religion, Class and Elections in Egypt (Problems of International Politics). Cambridge: Cambridge University Press.

Markey, P., \& Amrara, T. (2014). Tunisia's Islamist down but not out after election defeat. Reu- ters. Recuperado desde http://uk.reuters.com/ article/2014/10/30/uk-tunisia-election-idUKKBN0IJ1LF20141030

Monzón, I. (2015). Egipto reprime el aniversario de la revolución contra Mubarak. El País. Recuperado desde http://internacional.elpais.com/internacional/2015/01/25/actuali$\mathrm{dad} / 1422194515$ 571577.html

Ottaway, M. (2014). Democracy by Necessity. Foreign Affairs. Recuperado desde http://www. foreignaffairs.com/articles/142735/marina-ottaway/democracy-by-necessity

Putnam, R. (1993). Making Democracy Work: Civic Traditions in Modern Italy. Princenton: Princenton University Press.

Pew Research Center. (2014). Egyptian views of Leaders, Organizations, Institutions. En Egyptians Embrace Revolt Leaders, Religious Parties and Military, As Well. Pew Research Global Attitudes Project. Recuperado desde http://www.pewglobal.org/2011/04/25/chapter-2-views-toward-key-leaders-groups-and-institutions/

Pew Research Center. (2013). Pew Research Global Attitudes Project (Spring 2013). Recuperado desde www.pewglobal.org/2013/05/01/spring-2013-su

Reuters. (2014). Tunisia concede election defeat to secular party. Recuperado desde http:// www.reuters.com/article/2014/10/27/us-tunisia-election-idUSKBN0IG0C120141027

The Economist. (2014). Democratic Index 2013. Recuperado desde www.eiu.com/public/topical_ report.aspx?campaignid=Democracy0814

Trabelsi, M. (2012). La primavera árabe y el papel de los sindicatos, en Sindicatos autónomos egipcios: Desafios frente a los procesos de cambio político. Madrid: Agencia Española de Cooperación Internacional para el Desarrollo (AECID). 
UNICEF. (2013). Egypt. Recuperado desde http:// www.unicef.org/infobycountry/egypt_statistics.html

UNICEF. (2013). Literacy Rates. Recuperado de www.unicef.org.

Weaver, M., \& Whitaker, B. (2012). Egypt elections: latest results. The Guardian. Recuperado desde http://www.theguardian.com/world/middle-east-live/2012/may/25/egypt-elections-early-results-live

World Bank Group. (2014). Doing Business. Recuperado desde http://www.doingbusiness.org/ rankings

World Economic Forum. (2014). Competitiveness Rankings. Recuperado desde http://reports. weforum.org/global-competitiveness-report-2014-2015/rankings/

Zayed, A. (2014). The rise and fall of Egypt's Middle Class. Recuperado desde http://www.al-monitor.com/pulse/culture/2014/05/egypt-middle-class-rise-fall.html\#

Zakaria, F. (2013). Arab Spring's hits and misses. The Washington Post. Recuperado desde http://www.washingtonpost.com/opinions/ fareed-zakaria-arab-springs-hits-and-misses/2013/01/30/fc72dcc2-6b15-11e2-af537b2b2a7510a8_story.html

Zakaria, F. (2014). Can Arab countries be real democracies?. Global Public Square (CNN). Recuperado desde http://globalpublicsquare.blogs. cnn.com/2014/11/03/can-arab-countriesbe-real-democracies/ 